\title{
Numerical analysis of the tympanic membrane vibrations
}

\author{
V. Gylienė*, G. Janušas ${ }^{* *}$, G. Gylys ${ }^{* * *}$, Q. A. B. Nivault**** \\ *Kaunas University of Technology, Studentu 56, LT-51424 Kaunas, Lithuania, E-mail: virginija.gyliene@ktu.lt \\ **Kaunas University of Technology, Studentu 56, LT-51424 Kaunas, Lithuania, E-mail: giedrius.janusas@ktu.lt \\ ***Lithuanian University of Health Sciences, Department of Oto-Rhino-Laryngology, Eiveniu 2, LT-50009 Kaunas, \\ Lithuania,E-mail: gylysg@yahoo.com \\ ****Ecole Nationale d'Ingenieurs de Metz, 1 route d'Ars Laquenexy, FR-57078, Metz Cedex 3, France, \\ E-mail:q.nivault@enim.fr
}

cross $^{\text {ref }}$ http://dx.doi.org/10.5755/j01.mech.22.2.13565

\section{Introduction}

The human auditory system is a remarkable engineering design that is made up of complex geometries, many material properties and different dynamic responses to transform the acoustic input into an electrical output for humans to understand. The thing that is usually understood as ear, in reality, is only the small part of this sophisticated hearing organ. The human ear is composed of three parts: outer or external ear, middle ear and inner ear (Fig. 1.). The specific anatomical structure separating the outer and middle ear is the eardrum (Tympanic Membrane).

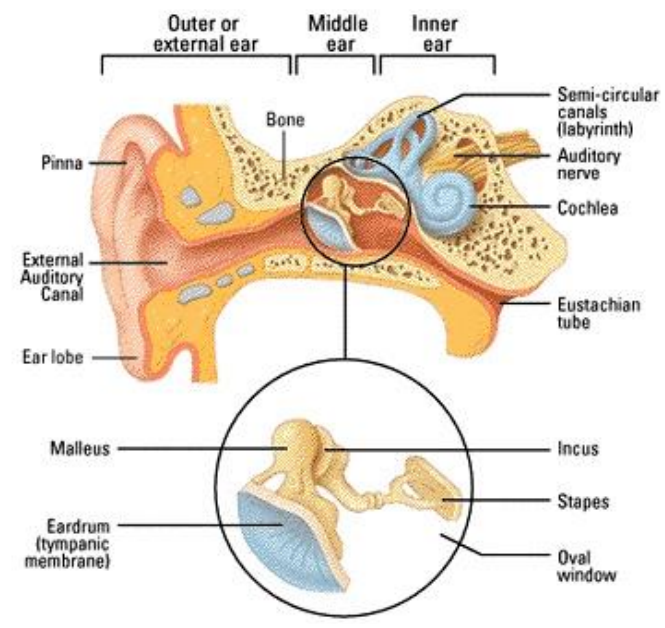

Fig. 1 The human ear system [1]

The investigation of Tympanic Membrane (TM) mechanics is important for understanding the acoustical and mechanical properties of the sound-path from the ear to the cochlea. The vibrations of the TM play a key role for sound transmission to the inner ear [2]. Finally, the tympanic membrane realises two functions [3]:

1. The transfer of sound waves to the ossicles;

2. The transmission of atmospheric pressure.

Ossicular anatomy and middle ear mechanics, besides its acoustical function, is as well influenced by nonacoustical loads, for example, atmospheric pressure variations. The ear works as a pressure receptor [4]. There are unresolved issues in understanding the tympanic membrane and its behaviour [2].

There are many diseases, which cause the tympanic membrane defects; the most common is the tympanic membrane perforation. For example, acute or chronic otitis media may result in defects of part or of the entire tympanic membrane [5]. Myringoplasty is considered the simplest reconstructive procedure of the middle ear [6]. In order to repair the perforation of TM, different types of grafts are used. After the myringoplasty, the healing TM becomes thicker, and the whole layer gets the same features as the normal TM. The changes that the tympanic membrane is overcoming after the myringoplasty are usually unclear and cannot be easily predicted. However, the TM restores the functional features. The disposed problematic is visualised in Fig. 2.

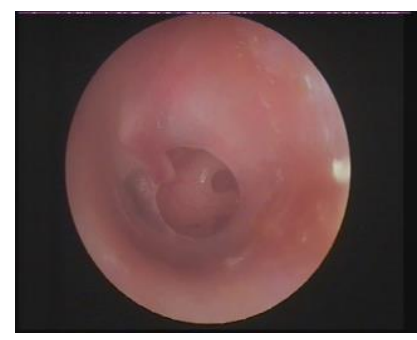

a

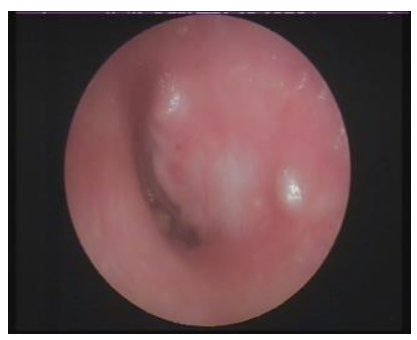

b
Fig. 2 Tympanic membrane: a - with perforation before the middle ear surgery; $b$ - thicker, but intact TM after the surgical repairing

The accurate model of the tympanic membrane, which simulates the acoustic-mechanical transmission, could improve the clinical surgical intervention [7]. For such kind of problems, the method of Finite Elements (FE) becomes an important tool, especially for the investigation of specific problems, such as [8, 9] middle ear acoustic-mechanical behaviour, the introduction of different kinds of implants and prosthesis.

Due to the above mentioned reasons, tympanic membrane was investigated in these ways:

- the composition of FE model and the frequency response analysis for healthy TM and TM with perforation according to the sound frequency,

- the analysis of the displacements of the intact TM and the TM after the operation of myringoplasty according to the predicted fixation place.

The frequency response analysis was provided for the intact ear and the TM with perforation. During the myringoplasty surgery, the geometrical changes of TM and its fixation is up to the surgeon. The study of the effect for the TM displacements according to the fixation position was 
performed. In both study cases, the dynamic load was taken into account.

\section{Finite element model of the tympanic membrane}

A variety of TM FE models can be found in the literature that differ in geometry, mesh/element, boundary condition and material properties [7].

In the tympanic membrane study from a mechanical point of view, the first objective was to create a geometrical model. Several groups have determined the geometric parameters of each middle ear component by using anatomical data or the data directly obtained from the measurements of temporal bones [7]. 3D Model of the middle ear assembly is presented in Fig. 3 (for more details, read [10]).

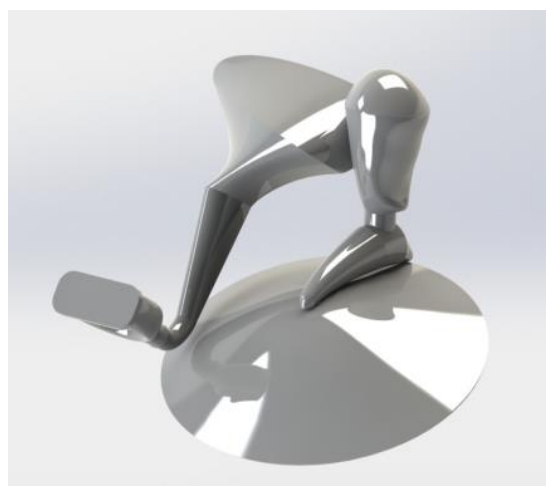

Fig. 3 The assembly of middle ear, performed in SolidWorks®2012 ([10])

Zhao [11] summarises the results of the investigation defining the geometries of the middle ear. The Table 1 provide the results of these findings and the geometries used in numerical simulations as well.

Table 1

Geometry configurations of the TM

\begin{tabular}{|l|c|c|}
\hline \multicolumn{1}{|c|}{ Parameter } & $\begin{array}{c}\text { Real } \\
\text { dimensions } \\
{[11]}\end{array}$ & Model \\
\hline $\begin{array}{l}\text { Diameter along the } \\
\text { manubrium, mm }\end{array}$ & $8.0 \div 10.98$ & 10 \\
\hline $\begin{array}{l}\text { Diameter perpendicular to } \\
\text { the manubrium, mm }\end{array}$ & $7.5 \div 9.22$ & 8 \\
\hline Height of the cone, mm & $1.42 \div 2.0$ & $0.91 \div 1.91$ \\
\hline Surface area, $\mathrm{mm}^{2}$ & $55.8 \div 79$ & $59.7 \div 71.2$ \\
\hline Thickness, mm & $0.05 \div 0.169$ & $0.1 \div 0.5$ \\
\hline
\end{tabular}

Concerning the FE modelling, different element types have been employed and the conclusion has been set that mainly shell elements are used for TM modelling [7], [11]. Material characterisation of the TM tissues still represents a debated issue due to the variety of tests, typically in vitro, but also in vivo [7]. The questions for the discussions are also: the thickness of tympanic membrane, the isotropic or orthotropic material characteristics, and the human or animal material characteristics. The majority of authors $[7,11,12]$ agree on the characteristics of the density and Poisson's coefficient. The value of $21 \mathrm{MPa}$ of Young's modulus was mentioned as giving the best results [13]. Ta- ble 2 summarises the mechanical material properties for linear elastic modelling.

Table 2

Mechanical properties of TM finite element model

\begin{tabular}{|l|c|}
\hline Density, $\mathrm{kg} / \mathrm{m}^{3}$ & 1200 \\
\hline Young's modulus, MPa & 21 \\
\hline Poisson's coefficient & 0.3 \\
\hline
\end{tabular}

TM being the soft tissue is modelled as viscoelastic material. Viscoelasticity is modelled by using a complex modulus in the frequency domain [13]:

$$
E(w)=E_{1}(w)+i E_{2}(w)=E_{1}(w)[1+i \eta(w)],
$$

here $w$ - the angular frequency; $E_{1}(w)$ - the storage modulus that accounts for the elastic portion (comparable to the conventional elastic Young's modulus); $E_{2}(w)$ - the loss modulus that accounts for the viscous portion; $\eta$ - the loss factor and $i$ - imaginary unit.

Several groups of authors [7, 14, 15] investigated the TM damping against the frequency range. The loss factor of $7.8 \%$ was more or less constant across the measured frequency range [14]. For the harmonic analysis, the loss factor of $7.8 \%$ has been selected as well for the adequacy of speech frequency range. Moreover, the stiffness damping parameter was set $\beta=10^{-4} \mathrm{~s}$ [7].

The most important frequencies for speech understanding are 500,1000, $2000 \mathrm{~Hz}$. This is the human voice frequency range, and the hearing is the most sensible in human ear to the frequency and amplitude modulation.

In the hearing system, the sound waves represent the dynamic pressure. The perceived sound consists of periodic acoustic pressure variations (sound pressure). The TM is moved by the sound pressure and works as sound receptor. The Sound Pressure Level (SPL) is the hearing feeling quantitative parameter. Following the mathematical relation, it can be used to define the pressure on the tympanic membrane:

$$
S P L=20 \log \left(\frac{p_{1}}{p_{0}}\right),
$$

where $p_{0}=2 \cdot 10^{-5} \mathrm{~Pa}$ is the reference sound pressure; $p_{1}$ is the pressure exerted on the TM.

Taking into account the physiological aspect that the TM is affected by pressure, the load of pressure was applied from the direction of external auditory canal. For the selection of the geometry of TM for frequency response analysis, the authors used findings of the previous hearing static analysis [16].

\subsection{The static analysis of tympanic membrane}

There are no data about the most common TM geometries in the human ear. The most typical three geometries of the eardrum were selected for the further analysis and created according to the angle of the cone as $10^{\circ}, 20^{\circ}$ and $30^{\circ}$. Perhaps the most expressive TM model is with angle of inclination of $30^{\circ}$ as presented in Fig. 4 . 


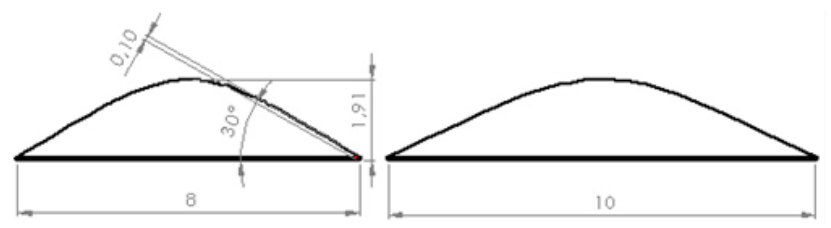

Fig. 4 The tympanic membrane model of angle of $30^{\circ}$

A uniform input pressure stimulus, corresponding to quiet room $(20 \mathrm{~dB})$ was applied to the lateral side of the TM as presented in Fig. 5. The same Fig. 5 presents the example of fixture on all periphery of TM.

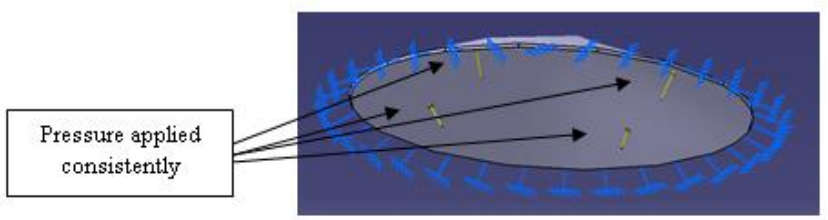

Fig. 5 The example of application of load on TM (shape of $30^{\circ}$ ) with all periphery fixture

During the myringoplasty surgery the tympanic membrane is becoming thicker, because the cartilage is fixed to cover the perforation. Also, the operation is due to close the hole of the TM. From that point of view, to study numerically the effect on TM displacements is of high importance. Generally, the surface area of perforation in TM is $6 \mathrm{~mm}^{2}$. Fig. 6 presents the numerical TM with perforation in the direction of manubrium.

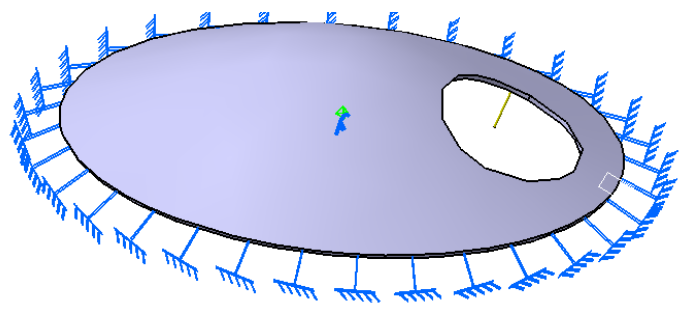

Fig. 6 Perforated TM for static FE analysis

Finally, summarising the presented aspects on intact and non-intact TM, the tympanic membrane displacement analysis was performed in the following way:

- the influence of the TM geometry to its displacements (TM fully clamped);

- the influence of the TM geometry to its displacements according to the clamping type.

For numerical FE modelling, the mesh size sensitivity analysis was performed, and the triangular element of $0.3 \mathrm{~mm}$ length was selected for the further numerical analysis on CatiaV5R21. The tympanic membrane movements are dependent on the morphology [3]. Usually, the cartilage material is used in myringoplasty. Three geometries, according to the angle $\left(10^{\circ}, 20^{\circ}\right.$ and $\left.30^{\circ}\right)$ of the cone of the TM, were selected for numerical analysis to test the influence of TM perforation in the case of TM thickness $0.1 \mathrm{~mm}$. The statement was proved in this analysis (Fig. 7).

From the Fig. 7 it is evident that the shape of TM of $10^{\circ}$ is the most sensitive and presents the highest displacements comparing with the other geometries. Comparing the displacement results of normal, intact TM without the hole, the displacements of eardrum of $10^{\circ}$ are $75 \%$ higher than the displacements in the case of eardrum of $30^{\circ}$.
Comparaison of displacements: TM intact/perforated

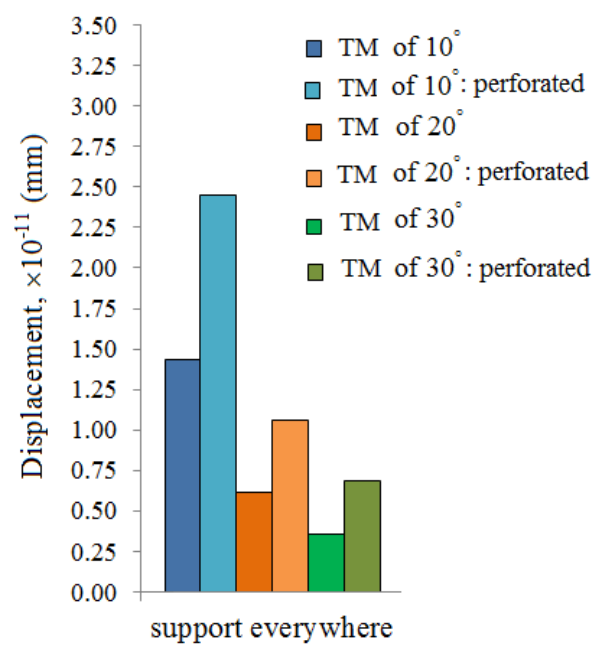

Fig. 7 TM displacements according to the shape: normal TM (thickness $0.1 \mathrm{~mm}$ ) without and with perforation

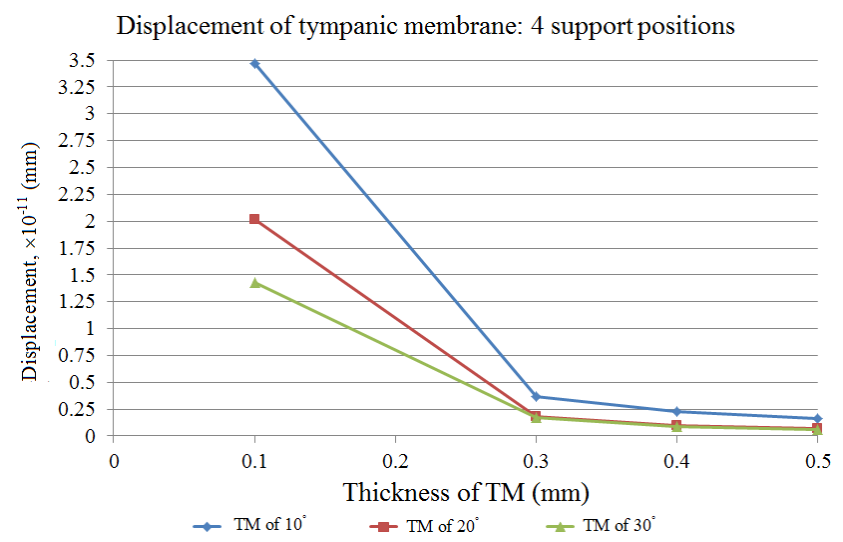

Fig. 8 TM displacements according to 4 fixture positions: thickness $0.1 \mathrm{~mm}$ - normal TM and thickness 0.3 , $0.4,0.5 \mathrm{~mm}$ after the myringoplasty

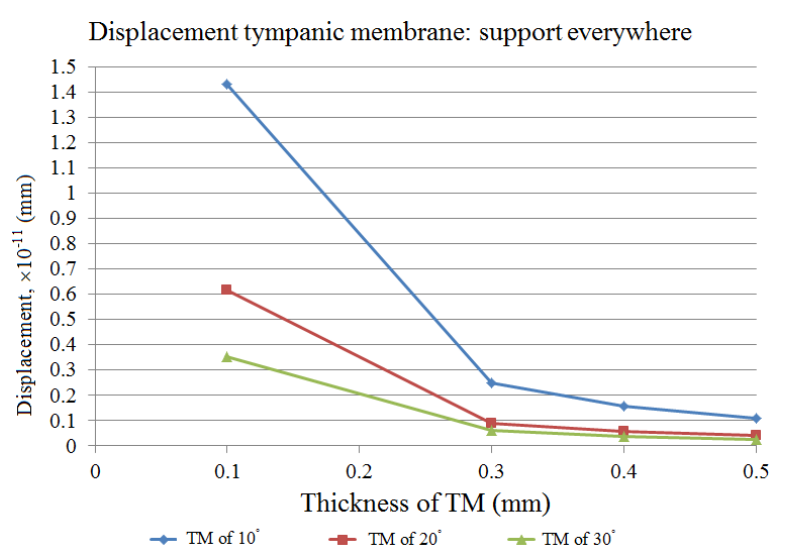

Fig. 9 TM displacements according to the complete fixture: thickness $0.1 \mathrm{~mm}$ - normal TM and thickness 0.3 , $0.4,0.5 \mathrm{~mm}$ after the myringoplasty

In the next stage, the numerical analysis was performed in order to define the TM fixation influence on the results of displacements in the case of healthy ear without perforation and in the case of the thicker TM (after operation). The cartilage slices prepared for the middle ear surgery that were performed in Lithuanian University Health Sciences are not thicker than $0.5 \mathrm{~mm}$. Other authors [5] provide the same suggestion about the cartilage preparation. 
Fig. 8 and Fig. 9 present the TM displacements according to the fixture of TM and the geometry (shape and thickness). The calculated data suggest that the most sensitive TM according the TM shape is the TM with the angle of the cone of $10^{\circ}$. Moreover, the results of static analysis show that the type of boundary conditions influences more than 2 times the TM displacements for all the geometries.

Summarising the findings from this static numerical study, it can be concluded that the intact TM (with common thickness $0.1 \mathrm{~mm}$ ) is as well sensitive to the boundary conditions. Moreover, the extreme influence is defined according to the shape of TM. Nevertheless, the results of Fig. 8 and Fig. 9 show that the reconstructed TM (becomes thicker) is less sensible to the boundary conditions. The

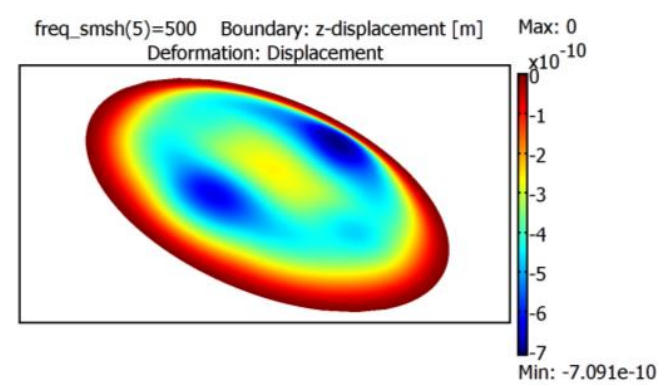

a

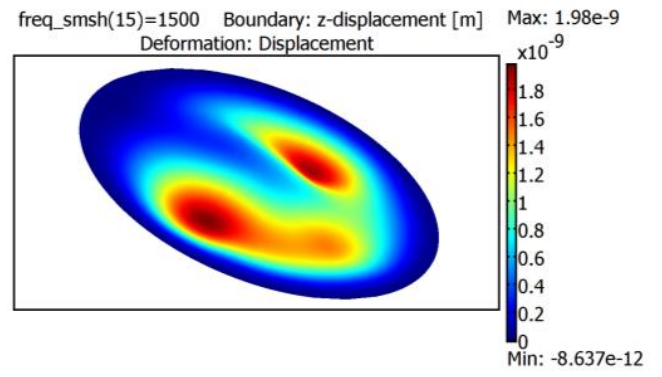

C statement could be made that the numerical model of the repaired TM could be simpler compared to the healthy TM numerical model, but this statement needs experimental validation.

\subsection{Tympanic membrane frequency response analysis}

The tympanic membrane frequency analysis was performed in Comsol Multiphysics 3.5a environment, considering the human voice frequency. From the previously summarized findings the selected geometry of TM was with angle of cone of $10^{\circ}$ with fully clamped periphery of TM to the ear canal. Table 3 presents the harmonic input pressure stimulus, applied to the lateral side of the TM.

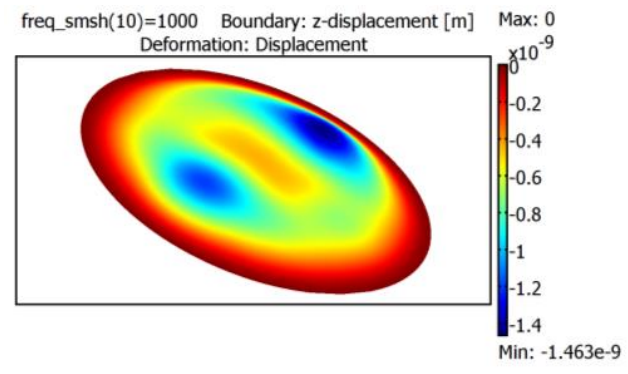

$\mathrm{b}$

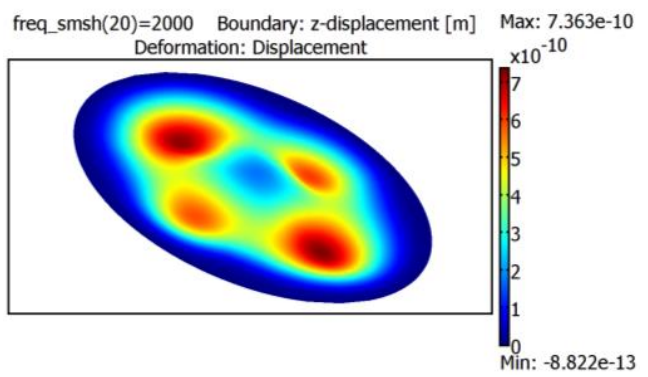

d

Fig. 10 Frequency response of intact tympanic membrane (the shape-angle of cone of $10^{\circ}$, thickness $-0.1 \mathrm{~mm}$ ): a - $500 \mathrm{~Hz}$; b - $1000 \mathrm{~Hz}$; - $1500 \mathrm{~Hz}$; - $2000 \mathrm{~Hz}$

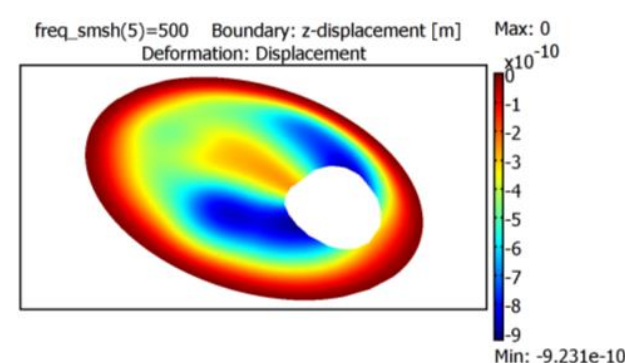

a

freq_smsh(15) $=1500 \quad$ Boundary: $z$-displacement $[\mathrm{m}] \quad$ Max: $3.951 \mathrm{e}-9$

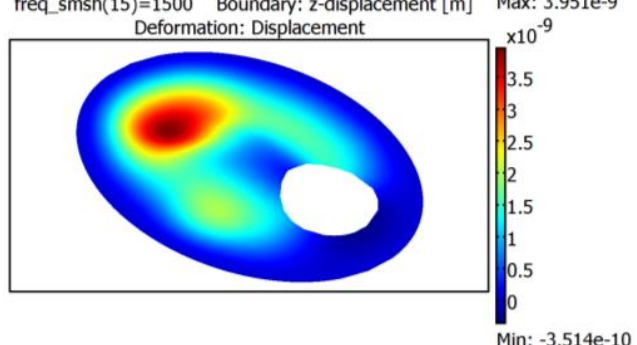

c

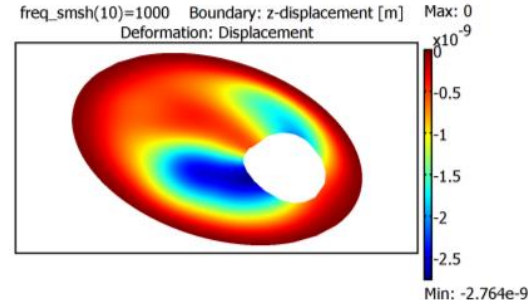

$\mathrm{b}$

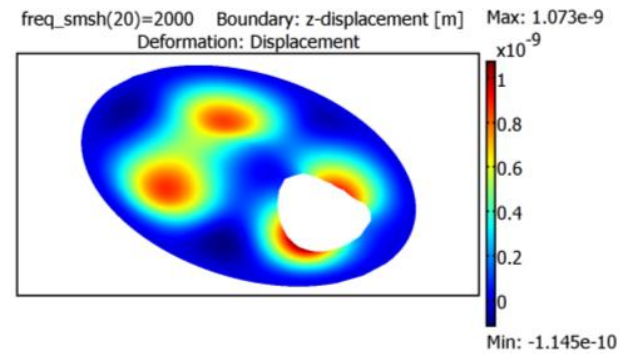

d

Fig. 11 Frequency response of tympanic membrane with perforation (the shape - angle of cone of $10^{\circ}$, thickness $-0.1 \mathrm{~mm}$, perforation area $-6 \mathrm{~mm}^{2}$ ): a - $500 \mathrm{~Hz}$; b $-1000 \mathrm{~Hz}$; $-1500 \mathrm{~Hz}$; $-2000 \mathrm{~Hz}$ 
Two sets of frequency response analysis were performed: for intact TM (Fig. 10) and TM with perforation (Fig. 11). In both study cases the thickness was $0.1 \mathrm{~mm}$.

Table 3

Harmonic input pressure stimulus

\begin{tabular}{|r|c|}
\hline Sound pressure level, $\mathrm{dB}$ & 45 \\
\hline Pressure transferred, Pa, found by & \\
Eq. (1) & 0.003557 \\
\hline & Frequency, Hz \\
& 500 \\
& 1000 \\
Wen Sound level $\Rightarrow$ & 1500 \\
& 2000 \\
\hline
\end{tabular}

To the best of our knowledge, there are no data about perforated tympanic membrane numerical modelling. The author De Greef [13] performed the identification of magnitude of vibrations and respectively the numerical simulation in the range of frequencies: $1 \mathrm{kHz}, 7 \mathrm{kHz}, 16 \mathrm{kHz}$. Numerical results were compared with experimental presented in [13]. The adequacy with accuracy of $10 \%$ was achieved comparing the formation and the magnitude of peaks. Two peaks are forming in the zone in pars tensa (against the malleus was unvalued in our model) comparing with experimental results [13].

\section{Conclusions}

In this work, the key aspect was the composition of linear elastic Finite Element model for harmonic analysis of intact tympanic membrane and tympanic membrane with central perforation. The numerical analysis of vibrations was performed in 500,1000,1500, $2000 \mathrm{~Hz}$ frequencies with the intensity of $45 \mathrm{~dB}$, trying to investigate how the intact and perforated tympanic membranes move. The acquired data in $1000 \mathrm{~Hz}$ fits the literature data.

From the numerical analysis we receive that maximum amplitude in healthy and perforated tympanic membrane differs two times in $1500 \mathrm{~Hz}, 70$ times in $2000 \mathrm{~Hz}$, while in 500 and $1000 \mathrm{~Hz}$ they move similarly. The numerical results fit the medical examinations that the central tympanic membrane perforation affects more high and middle range frequency than the low range one. In other words, the human with central perforation hears worse women speech or high frequency range.

Also performed static analysis shows that the tympanic membrane fixation place affects its movements after the myringoplasty according to the geometry of the

tympanic membrane: smaller angle of the cone means the higher sensitivity of TM displacements.

\section{References}

1. Deafness. National Cochlear Implant Users Association. Available at: http://www.nciua.org.uk/cochlear-implants/deafness/. [Revised: 11-06-2015].

2. Burkhardt, A.; Kirsten, L.; Bornitz, M.; Zahnert, T.; Koch, E. 2013. Vibration of the human tympanic membrane measured with OCT in a range between $0.4 \mathrm{kHz}$ and $6.4 \mathrm{kHz}$ on an ex vivo sample, Proc. SPIE 8805, Head and Neck Optical Diagnostics, 880507 (June 24, 2013). http://dx.doi.org/10.1117/12.2032549.

3. Vorwerk, U.; Hey, M.; Steinicke, G.; Begall, K. 1997. Methodic aspects of observations of rapid eardrum motions upon changes in static pressure (Valsalva maneuver) using digital high-speed video imaging, LaryngoRhino-Otol. 76(6): 341-346.

http://dx.doi.org/10.1055/s-2007-997439.

4. Huettenbrink, K.B. 2007. Clinical significance of stapedioplasty biomechanics: Swimming, diving, flying after stapes surgery, Otosclerosis and Stapes Surgery 65: 146-149.

http://dx.doi.org/10.1159/000098791.

5. Zahnert, T.; Bornitz, M.; K. B. Huttenbrink, K. B. Mechanic and acoustic properties of different materials for tympanic membrane reconstruction, Laryngo-RhinoOtol. 76(12): 717-723.

http://dx.doi.org/10.1055/s-2007-997513.

6. Klacansky, J. 2009. Cartilage myringoplasty, The Laryngoscope 119(11): 2175-2177.

http://dx.doi.org/10.1002/lary.20290.

7. Volandri, G.; Di Puccio, F.; Forte, P.; Carmignani, C. 2011. Biomechanics of the tympanic membrane, J. Biomech. 44(7): 1219-1236.

http://dx.doi.org/10.1016/j.jbiomech.2010.12.023.

8. Gentil, F.; Garbe, C.; Parente, M.; Martins, P.; Ferreira, A.; Jorge, R.N.; Santos, C.; Paco, J. Analysis of eardrum pathologies using the finite element method, J. Mech. Med. Biol. 14(3): 20.

http://dx.doi.org/10.1142/S0219519414500341.

9. Gentil, F.; Parente, M.; Martins, P.; Garbe, C.; Jorge, R.N.; Ferreira, A.; Tavares, J. M. R. S. 2011. The influence of the mechanical behaviour of the middle ear ligaments: a finite element analysis, Proc. Inst. Mech. Eng. Part H-J. Eng. Med., 225(H1): 68-76. http://dx.doi.org/10.1243/09544119JEIM783.

10. Branswyck, J.; Martin, R.; Gylienè, V.; Gylys, G. 2013. Introduction to hearing study, Conf. -12- Juniors Researchers: 2013, Electronic Source, ktu: 4.

11. Zhao, F.; Koike, T.; Wang, J.; Sienz, H.; Meredith, R. 2009. Finite element analysis of the middle ear transfer functions and related pathologies, Med. Eng. Phys. 31(8): 907-916.

http://dx.doi.org/10.1016/j.medengphy.2009.06.009.

12. Gan, R.Z.; Sun, Q.L., Feng, B.; Wood, M.W. 2006. Acoustic-structural coupled finite element analysis for sound transmission in human ear - Pressure distributions, Med. Eng. Phys. 28(5): 395-404.

http://dx.doi.org/10.1016/j.medengphy.2005.07.018.

13. De Greef, D.;Aernouts, J.; Aerts, J.; Cheng, J.T., Horwitz, R.; Rosowski, J.J.; Dirckx, J.J.J. 2014. Viscoelastic properties of the human tympanic membrane studied with stroboscopic holography and finite element modelling, Hear. Res. 312: 69-80. http://dx.doi.org/10.1016/j.heares.2014.03.002.

14. Aernouts, J.; Aerts, J.R.M.; Dirckx, J.J.J. 2012. Mechanical properties of human tympanic membrane in the quasi-static regime from in situ point indentation measurements, Hear. Res. 290(1-2): 45-54.

http://dx.doi.org/10.1016/j.heares.2012.05.001.

15. Zhang, X.; Gan, R.Z. 2012. Dynamic properties of human tympanic membrane based on frequency-temperature superposition, Ann. Biomed. Eng. 41(1): 205-214. http://dx.doi.org/10.1007/s10439-012-0624-2.

16. Nivault, Q.A.B. 2015. Study of the eardrum, Internal 
KTU-ENIM repport. 43.

V. Gylienè, G. Janušas, G. Gylys, Q.A.B. Nivault

\section{NUMERICAL ANALYSIS OF THE TYMPANIC MEMBRANE VIBRATIONS}

S u m m a r y

The intact tympanic membrane ensures a good hearing quality for humans. Sometimes, the tympanic membrane is perforated and because of that the hearing suffers. The tympanic membrane is reconstructed by covering the perforation with autogenous tissues that changes the tympanic membrane geometry, and the hearing is restored. During the hearing restoration operation, the surgeon influences the thickness of the tympanic membrane and the fixation type.

Nevertheless, it is still not clear why in some cases the hearing is deeply affected by small perforation. For this reason, a numerical model has been constructed for the intact and perforated tympanic membrane with common $6 \mathrm{~mm}^{2}$ perforation. The numerical analysis of vibrations was performed in 500, 1000, 1500, $2000 \mathrm{~Hz}$ frequencies with the intensity of $45 \mathrm{~dB}$ aiming to investigate how the healthy and perforated tympanic membranes move.

The acquired data at $1000 \mathrm{~Hz}$ correspond to the literature data. Numerical analysis shows that the maximum amplitude in intact and perforated tympanic membranes differs 2 times in $1500 \mathrm{~Hz}, 70$ times in $2000 \mathrm{~Hz}$, while in 500 and $1000 \mathrm{~Hz}$ they move similarly.

The analysis on how the tympanic membrane fixation place affects its movements after the myringoplasty and according to the geometry of the tympanic membrane was also performed.

Keywords: Tympanic membrane, eardrum, frequency response, myringoplasty, Finite Element Modeling (FEM).

Received November 03, 2015 Accepted March 15, 2016 